Archives de sciences sociales des religions

172 | octobre-décembre

Bulletin Bibliographique

\title{
Michel de Certeau, lecteur de Nicolas de Cues
}

Nicolas de Cues était-il un mystique?

Jocelyne Sfez

\section{(2) OpenEdition}

Journals

Édition électronique

URL : http://journals.openedition.org/assr/27220

DOI : $10.4000 /$ assr. 27220

ISSN : $1777-5825$

Éditeur

Éditions de l'EHESS

Édition imprimée

Date de publication : 1 octobre 2015

Pagination : 67-79

ISBN : 978-2-7132-2515-4

ISSN : 0335-5985

Référence électronique

Jocelyne Sfez, "Michel de Certeau, lecteur de Nicolas de Cues », Archives de sciences sociales des religions [En ligne], 172 | octobre-décembre, mis en ligne le 26 avril 2018, consulté le 03 mai 2019.

URL : http://journals.openedition.org/assr/27220 ; DOI : 10.4000/assr.27220

(C) Archives de sciences sociales des religions 


\section{Jocelyne Sfez}

\section{Michel de Certeau, lecteur de Nicolas de Cues Nicolas de Cues était-il un mystique?}

Dire que la sortie du deuxième tome de la Fable mystique de Michel de Certeau ${ }^{1}$ était attendue dans les milieux de la recherche cusaine serait un euphémisme. En 2005, Wilhelm Dupré, auteur de l'édition viennoise, dite du Jubilé, des principales œuvres de Nicolas de $\mathrm{Cues}^{2}$ et professeur émérite de philosophie de la religion à Nimègue, me demandait avec un désir dévorant, si l'on pouvait effectivement espérer qu'un jour paraisse ce texte annoncé en 2002 par François Dosse dans Michel de Certeau, le marcheur blessé ${ }^{3}$. François Dosse précisait dans son ouvrage, sur la base d'une lettre de Michel de Certeau à Pierre Nora du 19 août 1984, qu'avant sa disparition, en 1986, l'auteur de la Fable mystique avait programmé un deuxième volume, qui devait s'intituler «La Fable mystique : une science expérimentale ", et être composé de trois chapitres principaux, dont le premier serait consacré à Nicolas de Cues et intitulé "Nicolas de Cues : le regard fondateur ", les deux autres portant respectivement sur Thérèse d'Avila et Jean de la Croix.

Il ne faisait aucun doute, pour nous cusains, que ce chapitre inaugural et le titre de "science expérimentale » employé pour le projet futur et renvoyant à une notion si importante chez le Cusain montraient assez le rôle décisif que Michel de Certeau entendait attribuer à Nicolas de Cues dans la mystique chrétienne et nous nous en réjouissions autant que nous déplorions encore l'absence de la publication. Je ne sais si Wilhelm Dupré en connaît la parution aujourd'hui, mais j'ai déposé un exemplaire de l'ouvrage à l'Institut für Cusanus-Forschung : le maître ouvrage de Michel de Certeau y a assurément toute sa place et renouvellera, j'en suis sûre, l'approche de la mystique dans l'œuvre du Cusain, en lui donnant probablement une assise un peu plus solide, scientifiquement parlant, que celle qu'elle possède bien souvent encore actuellement.

1. Michel de Certeau, La Fable mystique II, XVI ${ }^{e}-X V I I^{e}$ siècle (éd. établie et présentée par Luce Giard), Paris, Gallimard, "Bibliothèque des Histoires ", 2013.

2. Nikolaus von Kues, Philosophisch-theologischen Schriften (éd. Leo Gabriel) (trad. et commenté par Dietlind et Wilhelm Dupré), Vienne, Herder, 1964, 3 tomes.

3. François Dosse, Michel de Certeau. Le marcheur blessé, Paris, La Découverte, 2002. 
Il n'est déjà pas trop de faire remarquer que, lorsque paraît le premier volume de la Fable mystique, Nicolas de Cues n'est pas étudié en France. Ses œuvres ne sont pas traduites en français. Il y a bien eu la thèse de Maurice de Gandillac et un volume d'extraits choisis, parus respectivement en 1941 et 1942 chez Aubier Montaigne ${ }^{4}$, mais ces livres ne sont plus disponibles dans le commerce, et seule une épouvantable traduction de la Docte Ignorance de 1930 circule, republiée chez Trédaniel en $1979^{5}$. L'Université de Sherbrooke a bien publié successivement la Concordance catholique ${ }^{6}$ et la Paix de la fo ${ }^{7}$, mais cela se passe outreAtlantique. Outre-Rhin, l'édition scientifique et critique de l'Akademie der Wissenschaften de Heidelberg est en chantier depuis les années 30, et sont déjà parus de très nombreux ouvrages du Cusain, dont la Docte Ignorance, les Conjectures, la Chasse de la sagesse, l'Apologie de la Docte Ignorance, la Paix de la foi, etc. ${ }^{8}$. Rudolf Haubst crée en 1979 l'Institut für Cusanus-Forschung pour éditer l'ensemble des presque 300 sermons. Maurice de Gandillac publiera encore en 1985 certaines lettres du Cusain aux moines de Tegernsee sur la docte ignorance et le Jeu de la Boule 9 .

Mais c'est après la Fable mystique $\mathrm{I}^{10}$. Surtout, Agnès Minazzoli publie en $1986^{11}$ sa remarquable traduction du De visione Dei seu de icona, qu'elle dédie à Michel de Certeau, dont on sent bien, à la lecture de la préface, qu'il a habité son travail de chercheuse et de traductrice ${ }^{12}$. Celui-ci trouve écho chez l'historien de l'art Daniel Arasse ${ }^{13}$. Il faut ensuite attendre le début des années 1990 pour que le travail d'édition des textes cusains s'amorce pleinement en France ${ }^{14}$. En

4. Maurice de Gandillac, La philosophie de Nicolas de Cues, Paris, Aubier-Montaigne, "Philosophie de l'esprit », 1941 ; Nicolas de Cues, Euvres choisies (trad. Maurice de Gandillac), Paris, Aubier-Montaigne, "Bibliothèque philosophique », 1942.

5. Nicolas de Cues, De la docte ignorance (introd. Abel Rey), Paris, Guy Trédaniel, Éditions de la Maisnie, 1979.

6. Nicolas de Cues, La Concordance catholique (trad. Roland Galibois), Sherbrooke, Centre d'Études de la Renaissance, Université de Sherbrooke, 1977.

7. Nicolas de Cues, La Paix de la foi (trad. Roland Galibois), Sherbrooke, Centre d'Études de la Renaissance, Université de Sherbrooke, 1977.

8. On trouvera un écho français de ces publications dans Maurice de Gandillac, "Actualité de Nicolas de Cues ?", Bulletin de la Société française de philosophie, 60/1, 1966, et dans Louis-Jacques Bataillon et Christoph von Schönborn, "Connaissance de Nicolas de Cues ", Revue des sciences philosophiques et théologiques, 56/1, 1972, p. 63-77.

9. Nicolas de Cues, Lettres aux moines de Tegernsee sur la docte ignorance. Du Jeu de la boule, Paris, OEIL, "Sagesse chrétienne ", 1985.

10. Michel de Certeau, La Fable mystique I, XVI" $-X V I I^{e}$ siècle, Paris, Gallimard, "Bibliothèque des Histoires ", 1982 [republié : Paris, Gallimard, "Tel », 1987].

11. Nicolas de Cues, Le tableau ou la vision de Dieu (trad. Agnès Minazzoli), Paris, Le Cerf, coll. "La nuit surveillée ", 1986 [rééd. : Paris, Les Belles Lettres, "l'Ymagier », 2012].

12. Cf. également Agnès Minazzoli, La première ombre. Réflexion sur le miroir et la peinture. Paris, Minuit, 1986.

13. Cf. Daniel Arasse, On n'y voit rien. Descriptions, Paris, Folio Essais, 2003 (Denoël, 2000).

14. Sur le renouveau des traductions et des études cusaines en France, $c f$. Jocelyne Sfez, "Actualité de Nicolas de Cues. Publications francophones récentes ", Études philosophiques, Paris, PUF, 2013/4, p. 575-599. 
Allemagne, le commentaire de Certeau est reçu dans le milieu cusain, d'une part par ceux qui, comme Dupré ou Sengers ${ }^{15}$, inscrivent Nicolas de Cues dans l'héritage de la mystique rhénane, d'autre part par ceux qui étudient l'influence du Cusain sur le développement de la philosophie du XX ${ }^{\mathrm{e}}$ siècle. C'est notamment le cas de Johannes Hoff qui a examiné l'apport cusain aux pensées de Derrida et de Certeau ${ }^{16}$. Michel de Certeau s'avère dès lors, au-delà de Maurice de Gandillac, à la fois comme un pionnier dans la redécouverte en France de Nicolas de Cues - et ce, dès avant la parution de la Fable mystique $\mathrm{I}^{17}$ - et comme un commentateur reconnu de l'œuvre cusaine.

Luce Giard, dans l'édition du volume qu'elle nous livre aujourd'hui, revient dans sa présentation sur la teneur et la composition de ce deuxième opus. Dans la lettre de Certeau qu'elle cite, du 25 août 1984 à Michael B. Smith, le volume semble avoir pris de l'ampleur par rapport à la lettre du 19 août adressé à Pierre Nora, puisqu'il s'étend maintenant de Nicolas de Cues à Pascal ${ }^{18}$. Le premier chapitre, effectivement consacré à Nicolas de Cues, et le plus long de tout le volume (plus de 70 pages), est pour nous très important, car il est pour une bonne partie inédit, bien qu'il reprenne un article d'une quinzaine de pages, "Nicolas de Cues : le secret d'un regard ", que Michel de Certeau avait publié en $1984^{19}$ dans la revue Traverses et où il avait déjà analysé la préface du De visione Dei, qu'il appelle de son nom alternatif de De icona.

Le chapitre publié aujourd'hui dans La Fable mystique II, davantage contextualisé, réinscrit l'ouvrage dans l'ensemble de l'œuvre cusaine et tente d'en dégager les conséquences pour la conception cusaine du politique, en particulier à

15. Cf. Hans-Georg Sengers, "Mystik als Theorie bei Nikolaus von Kues ", Gnosis und Mystik in der Geschichte der Philosophie (éd. Peter Koslowski), Zurich-Munich, ArtemisVerlag, 1988, p. 111-134.

16. Cf. en particulier, Johannes Hoff, "Philosophie als performative Pratik. Spuren cusanischen Denkens bei Jacques Derrida und Michel de Certeau ", Cusanus-Rezeption in der Philosophie des 20. Jahrhunderts (éd. Klaus Reinhardt, Harald Schwaetzer). Ratisbonne, Roderer, "Philosophie Interdisziplinär» 13, 2005, p. 93-119; «Berührungspunkte. Ein Tria$\log$ zwischen Jacques Derrida, Nicolaus von Kues und Michel de Certeau ", Michel de Certeau. Geschichte-Kultur-Religion (éd. Marian Füssel), Constance, Uvk Verlagsgesellschaft, 2007 : 17 342 ; Kontigenz, Berührung, Überschreitung : zur philosophischen Propädeutik christlicher Mystik nach Nikolaus von Kues, Munich, Karl Alber, 2007.

17. Des remarques incidentes sur l'œuvre du Cusain apparaissent en effet dès 1963 dans l'œuvre de Certeau, avant les articles de Gandillac et de Bataillon-Schönborn (cf. supra, n. 8). Cf., par exemple, l'article "France ", "V. Le XVI siècle. 2. La Réforme dans le catholicisme ", Dictionnaire de spiritualité ascétique et mystique, Paris, t. 5, 1963, col. 896-910, repris dans le volume établi par Luce Giard; Michel de Certeau, Le Lieu de l'autre. Histoire religieuse et mystique, Paris, Seuil/Gallimard, "Hautes études ", 2005, p. 135-153, p. 139 et p. 142, ou encore, repris dans le même volume, p. 45-57, p. 51, l'article " Histoire et mystique ", Revue d'histoire de la spiritualité, t. 48, 1972, p. 69-82.

18. Luce Giard, "Présentation », Michel de Certeau, La Fable mystique II, op. cit., 7-17, p. $12-13$.

19. Michel de Certeau, "Nicolas de Cues : le secret d'un regard ", Traverses, Paris, CCI, 30-31, p. 70-85. 
partir de sa mise en perspective avec le De pace fidei, écrit par le Cusain à la même époque.

Ce qui étonne au premier regard le spécialiste cusain, c'est cette exceptionnelle connaissance de Nicolas de Cues, dans toute l'extension de son œuvre. Dans le deuxième volume de la Fable mystique, Michel de Certeau restitue d'entrée de jeu le contexte historique et culturel précis dans lequel fut rédigée la Vision de Dieu, qu'il relie immédiatement à la Paix de la foi, ou, comme Certeau traduit plus justement, "à la foi instauratrice de la paix ${ }^{20}$ ». Tous les deux traitent de la question de la vision ${ }^{21}$ : «Qu'est-ce que "voir" ? Comment une "vision" peutelle donner jour à un nouveau monde ? " Question cruciale, brûlante en 1453, lorsque le monde chrétien est menacé de destruction. Michel de Certeau donne ses sources : Edmond Vansteenberghe et son énorme dossier, inégalé, concernant les diverses polémiques suscitées par la Docte ignorance et son principe fondateur de coïncidence des opposés, écrite en 1440, et les diverses pièces à conviction dans ces débats ${ }^{22}$, écrits qui jalonnent la ou les disputes.

Polémiques et textes éclairent précisément le caractère difficultueux du texte cusain, en plaçant au centre son rapport à la théologie mystique et à la mystique je souligne que je distingue délibérément les deux. Les points de vue adoptés par les contradicteurs contemporains du Cusain sont diamétralement opposés. Dans un premier temps, on lui reprochera son anti-intellectualisme et un certain eckhartisme ; c'est la position de Johannes Wenck dans son De ignota litteratura en 1442-43 ; en réponse, Nicolas rédigera en 1449 son unique texte polémique : l'Apologie de la Docte ignorance ${ }^{23}$. Dans un deuxième temps, on reprochera au Cusain un excès d'intellectualisme dans son approche de la nescience, compromettant par l'étalage d'un savoir spéculatif l'accès à Dieu par pur amour ; c'est la position de Vincent d'Aggsbach ${ }^{24}$, persuadé de l'incompatibilité de la foi avec tout langage et toute connaissance et s'opposant dès lors à l'idée d'une

20. Michel de Certeau, La Fable mystique II, op. cit., p. 52.

21. La question de la vision était déjà référée au Cusain dans le premier volume de la Fable mystique, op. cit., p. 76-77.

22. Edmond Vansteenberghe, Autour de la docte ignorance, Münster, Aschendorff, «Beiträge zur Geschichte der Philosophie des Mittelalters ", XIV, 1915.

23. Cf., par exemple, Nicolas de Cues, Trois traités sur la docte ignorance et la coïncidence des opposés (éd. Francis Bertin), Paris, Le Cerf, "Sagesses chrétiennes ", 1991, p. 9 - et non, comme le donne à tort Michel de Certeau avec Gerhard Ritter, en 1448 (Michel de Certeau, La Fable mystique II, op. cit., p. 54). Il y a une faute d'impression dans la note 5 de cette page, concernant la datation du De ignota litteratura en 1542 et 1548 . Il faut évidemment lire 1442 et 1448 .

24. Cf., par exemple, la réponse indirecte de Nicolas de Cues à Vincent d'Aggsbach, dans sa lettre du 14 septembre 1453 à Gaspard Aindorffer, in Nicolas de Cues, Lettres aux moines de Tegernsee sur la docte ignorance (éd. Maurice de Gandillac), op. cit., p. 26-31; le texte de Vincent d'Aggsbach demandant à Nicolas des précisions sur la lecture gersonienne de Denys est perdu (n. 2, ibid, p. 52). 
coïncidence des contraires dans la docte ignorance ${ }^{25}$; en réponse Nicolas rédigera successivement en 1453 ses Compléments mathématiques et théologiques, la Vision de Dieu ou l'image, et encore le Béryl en 1458. Le De icona constitue l'épicentre de l'analyse de Certeau.

Il s'agit bien là du cœur du problème concernant la réception de l'œuvre cusaine, problème que Michel de Certeau, en une certaine mesure, reconduit dans son propre commentaire : reprenant en partie son concept de docte ignorance à la Théologie mystique de Denys l'Aréopagite, l'œuvre cusaine peut être mobilisée alternativement par les tenants des deux voies, celle de la connaissance et celle de l'amour unitif, celle de l'intelligence et celle de l'affectivité dans l'ascension vers Dieu ${ }^{26}$. Mais, s'il est indéniable que le Cusain a fréquenté assidûment le texte dionysien, une de ses sources majeures, dont il possédait pourtant les grands commentaires de la tradition, des Victorins à Thomas d'Aquin et Albert le Grand, en passant par Hugo de Balma ou Jean Gerson, il serait erroné d'y réduire son concept de docte ignorance, et plus encore son principe de coïncidence des opposés qui vaut, quoi qu'en disent les tenants d'une mystique cusaine, bien audelà ou en deçà de la sphère divine ${ }^{27}$. Ce qui est novateur chez le Cusain, c'est précisément que la coïncidence des opposés n'est pas affirmée seulement en Dieu, renvoyant à son inconnaissabilité et à son indicibilité et conduisant à l'épanouissement d'une théologie négative ou mystique, comme étant la plus vraie et la seule possible. La coïncidence des opposés est aussi affirmée dans toute assertion en notre esprit, à un certain degré d'approfondissement de notre connaissance, et aussi dans le monde ${ }^{28}$.

À cet égard, il n'est pas indifférent que, dans le premier volume de la Fable mystique, après avoir souligné l'essentialité du corpus dionysien ${ }^{29}$ dans l'émergence et l'émancipation de la "science mystique» ou de la mystique au $\mathrm{XVI}^{\mathrm{e}}$ siècle, Michel de Certeau enchaîne : "toute une tradition de commentaires [du corpus dionysien] se capitalise et se renouvelle : au XvI ${ }^{\mathrm{e}}$ s. on édite ceux de Jean Eck (1519), Denys le Chartreux (1536, 1556), Marsile Ficin (1538, 1561),

25. Sur ce point, il est donc difficile de suivre Michel de Certeau, lorsque, dans le premier volume de la Fable mystique, n. 121, p. 145, s'appuyant sur Edmond Vansteenberghe, Autour de la docte ignorance..., op. cit., p. 208, il qualifie Vincent d'Aggsbach de "cusain extrémiste et prophète de la Docte ignorance " qui « refusait même d'assimiler à la théologie mystique la contemplation, à cause de la connaissance qu'elle comportait encore ".

26. Sur ces deux voies et leur compréhension, cf. notamment Ysabel de Andia, Denys l'Aréopagite : tradition et métamorphoses, Paris, Vrin, 2006.

27. Sur l'origine du concept de "docte ignorance » et du principe de coïncidence des opposés, cf. en particulier la n. 5 dans Jocelyne Sfez, L'Art des conjectures de Nicolas de Cues, Paris, Beauchesne, p. 64 sq.

28. Cf., par exemple, Nicolas de Cues, La docte ignorance, II, 9, 156: «Le centre du monde coïncide avec la circonférence. »

29. Michel de Certeau, La Fable mystique I, op. cit., p. 121 : « [...] depuis le XIII siècle [...] et jusqu'à Bérulle, Denys l'Aréopagite [est] la référence dominante. » 
Nicolas de Cues (1565), Thomas d'Aquin (1588), etc. ${ }^{30}$ "Cela doit amener plusieurs remarques : 1 . si la philosophie cusaine est tributaire de sa réflexion sur l'Aréopagite, je ne connais en celle-ci, contrairement à celle de Thomas d'Aquin par exemple, aucun commentaire de l'œuvre du Pseudo-Denys, à proprement parler - car le commentaire est un genre d'écriture au Moyen Âge ; 2. l'édition de Bâle en 1565 des œuvres cusaines reprend sans y ajouter rien d'autre que des erreurs l'édition de Paris en 1514 par Lefèvre d'Étaples : la Parisiana, dont nous avons fêté l'année dernière les cinq cents ans, fut l'édition de référence des œuvres cusaines jusqu'au $\mathrm{XX}^{\mathrm{e}}$ siècle et elle assura avant la fin du $\mathrm{XVI}^{\mathrm{e}}$ siècle la dissémination de la pensée de Nicolas dans toute l'Europe ${ }^{31}$ dans les cercles savants, en particulier ceux de la science moderne naissante; 3 . tout cela ne veut évidemment pas dire que la réflexion du Cusain n'a pas contribué à la constitution d'une mystique, mais peut-être seulement précisément pour ses élaborations dans le De icona, qui connut comme le De pace fidei de multiples éditions et traductions isolées tout au long $\mathrm{du} \mathrm{XVI}{ }^{\mathrm{e}}$ et $\mathrm{du} \mathrm{XVII}^{\mathrm{e}}$ siècle, éditions que connaît manifestement Michel de Certeau ; 4. enfin, il faut se garder de trouver dans un intérêt immédiat pour la Théologie mystique du Pseudo-Denys un indice certain d'une pensée mystique: dira-t-on de Thomas d'Aquin ou d'Albert le Grand, par exemple, qu'ils sont des mystiques parce que Denys occupe dans leur réflexion une place véritable au point qu'ils en rédigent un commentaire ? Évidemment, non, et Michel de Certeau ne dit rien de tel.

Je souligne seulement ces points parce qu'il serait facile d'utiliser la Fable mystique comme élément de preuve pour accréditer la thèse d'un Nicolas de Cues mystique, thèse très en vogue dans les milieux cusains ${ }^{32}$, mais thèse qui

30. Michel de Certeau, La Fable mystique I, op. cit., p. 141.

31. Cf. Stephan Meier-Oeser, Die Presenz des Vergessen. Zur Rezeption der Philosophie des Nicolaus Cusanus, vom 15. bis zum 18. Jahrhundert, Münster, Aschendorff, "Buchreihe der Cusanus-Gesellschaft », X, 1989.

32. Outre l'article, déjà cité n. 15 , de H. G. Sengers, le lecteur pourra à propos de cette conception se rapporter à : Wilhelm Baum, "Nikolaus von Kues und die spätmittelalterliche Mystik in Österreich ", in Konziliarismus und Humanismus : Kirchliche Demokratisierungsbetrebungen im Spätmittelalterlichen Österreich (éd. Wilhelm Baum), Vienne, Turia+Kant, 1996, p. 112-137; Werner Beierwaltes, "Mystische Elemente im Denken des Cusanus ", in Deutsche Mystik im abendländischen Zusammenhang (éd. Walter Haug, Wolfram Schneider-Lastin), NY, De Gruyter, 1998, p. 425-448 ; Tilman Borsche, "Strategien zur Überwindung von Gegensätzen. Mystik und Irenik im Werk und Leben des Nikolaus von Kues ", in Toleranz und Religion (éd. W. Brändle, G. Leder, D. Lüttge), Hildesheim, Olms, 1996, p. 69-80 ; Walter-Andreas Euler, "Der Blick über die Mauer. Mystische Motive im Denken des Nikolaus von Kues ", in Die Realität des Inneren. Der Einfluß der deutschen Mystik auf die deutsche Philosophie (éd. Gerhard Stamer). Amsterdam-NY, Rodopi, 2001, 72-85 ; Alois Maria Haas, "... Das letzte unserer Sehnsüchte Erlangen ", Nikolaus von Kues als Mystiker, Trèves, Paulinus, «Trierer Cusanus Lecture ", 14, 2008 ; Alois Maria Haas, Mystik im Kontext, Munich, Fink, 2004 ; Alois Maria Haas, Deum mistice videre... in caligine coincidencie. Zum Verhältnis Nikolaus von Kues zur Mystik, Bâle/Francfort, "Vorträge der Aneas-Silvius-Stiftung an der Universität Basel ", 1989 ; Rudolf Haubst, "Christliche Mystik im Leben und Werk des Nikolaus von Kues ", in Die Bedeutung der Mystik für die Kirche (éd. Ulrich Dobhan et al.), Trèves, "Trierer 
peut s'avérer très problématique. De la même façon, c'est avec beaucoup de circonspection que nous voyons Michel de Certeau souligner son expérience rhénane et l'influence de Maître Eckhart qualifié d' «instaurateur » ${ }^{33}$.

Il est inutile de revenir en détail sur cette dernière question, qui a été traitée de manière décisive par Kurt Flasch ${ }^{34}$, historien de la philosophie médiévale et spécialiste à la fois de Maître Eckhart et de Nicolas de Cues. Je me contenterai donc de rappeler l'objection massive de cet éminent médiéviste à la qualification de mystique pour Maître Eckhart. C'est, dit Flasch, un anachronisme, dont "l'usage est apparu alors que les écrits latins d'Eckhart étaient encore inconnus et que les historiens croyaient voir en la "mystique" une voie alternative à la "scolastique" durant le Moyen Âge ".

En ce qui concerne Maître Eckhart, cette qualification de "mystique » est due aussi, en partie, à sa condamnation par l'Église romaine comme « semailles du diable » ${ }^{35}$, et, en partie, à sa reprise déformée chez Tauler et Suso, qui, eux, constituent vraiment des tenants de la mystique rhénane. Il n'est pas aussi aisé de dire la même chose de Maître Eckhart, maître en théologie, qui écrit en toutes lettres dans son Commentaire latin de l'Évangile de Jean, $\mathbb{S} 2-3$, qu'il adresse naturellement aux universitaires : «2. [...] l'auteur se propose, comme dans tous ses écrits, d'expliquer par les raisons naturelles des philosophes les affirmations de la sainte foi chrétienne et de l'Écriture dans les deux Testaments. [...] 3. [...] l'intention de cette œuvre est de montrer comment les vérités des principes, des conclusions et des propriétés des choses de la nature sont clairement indiquées dans ces mêmes mots de l'Écriture sainte que l'on interprète au moyen de ces réalités naturelles ${ }^{36}$." Maître Eckhart s'adresse certes ici en latin au clerc,

Scripte ", 1983 ; Klaus Reinhardt, "Negative Theologie und Koinzidenz der Gegensätze bei Nikolaus von Kues und in der rheinischen Mystik (Heinrich Seuse) ", in Nikolaus von Kues : ein bewundertswerter historischer Brennpunkt (éd. Klaus Reinhardt, Harald Schwaetzer), Ratisbonne, Rodorer, 2008, p. 21-39; Klaus Reinhardt, Harald Schwaetzer, "Mystik und Kirchenreform bei Nikolaus von Kues ", in Die Kirchenkritik der Mystiker, II (éd. Mariano Delgado, Gotthard Fuchs), Stuttgart, Kolhammer, 2005, p. 39-57. Christiane Bacher achève actuellement une thèse prometteuse sur les rapports entre mystique et science expérimentale à l'université de Hildesheim sous la direction de Tilman Borsche: Philosophische Waagschalen. Experimentale Mystik bei Nikolaus von Kues mit Ausblick auf die Existenzphilosophie.

33. Michel de Certeau, La Fable mystique I..., op. cit., p. 29.

34. Je renvoie donc à l'article fondamental de Kurt Flasch, « Meister Eckhart. Versuch, ihn aus dem mystischen Strom zu retten ", in Gnosis und Mystik in der Geschichte der Philosophie (éd. Peter Koslowski), op. cit., p. 94-110. Kurt Flasch a livré une présentation philosophique de Maître Eckhart particulièrement éclairante dans son ouvrage paru initialement en 009 en allemand et dont la $2^{\mathrm{e}}$ édition (2010) a été traduite en français: Maître Eckhart, philosophe du christianisme (trad. Catherine König-Pralong), Paris, Vrin, «Bibliothèque des philosophies ", 2011.

35. Cf. Kurt Flasch, Maître Eckhart, op. cit., p. 27.

36. Maître Eckhart, Commentaire de l'Évangile selon Jean (trad. Alain de Libera, Édouard Wéber et Émilie zum Brunn), Paris, Le Cerf, 1989, t. 6, p. 27-29 (Lateinische Werke III. Stuttgart, Kohlhammer, p. 4). Cf. Kurt Flasch, Maître Eckhart, op. cit., p. 48 sq. 
ailleurs en allemand à l'idiota qui, comme Ruedi Imbach l'a montré ${ }^{37}$, n'a rien, au Moyen Âge en tout cas, d'un simple d'esprit illuminé - mais avec le même projet rationnel et philosophique. C'est de l'universalité de l'homme que parle Maître Eckhart, non d' "espaces irréductibles à la spéculation théologienne et professionnelle ${ }^{38}$ " ou d'expériences subjectives, de visions ou d'extases au sens d'abîme de l'intellect. C'est bien là peut-être le drame pour l'Église de son temps.

Or, contrairement à ce qu'Alain de Libera et Frédéric Nef suggèrent dans "Le discours mystique. Problèmes d'histoire et de méthode ${ }^{39}$ ", c'est précisément parce qu'il n'a pas une conception anhistorique que Michel de Certeau étudie de façon délibérément délimitée le moment mystique du XVI ${ }^{\mathrm{e}}$ et XVII ${ }^{\mathrm{e}}$ siècle, celui de la mystique moderne. Dans la mesure même où Certeau constitue historiquement son objet ${ }^{40}$ en repérant la transformation de l'adjectif en substantif tout en en élucidant les motifs, la démarche de Certeau est respectueuse des auteurs et des traditions qu'elle aborde ou réfléchit, même lorsqu'ils ne sont pas au centre de son propos ${ }^{41}$. C'est ainsi que j'interprète ses remarques contenues vis-à-vis de Maître Eckhart et de la mystique rhénane. Une prudence, que l'on

37. Cf. Ruedi Imbach, Catherine König-Pralong, Le défi läqque, Paris, Vrin, "Conférences Pierre Abélard », 2013. Cette analyse est fort différente de celle menée par Michel de Certeau dans La Fable mystique I, op. cit., p. 321 sq. Mais c'est que, là encore, l'objet en est historiquement déterminé.

38. Michel de Certeau, La Fable mystique I..., op. cit., p. 145.

39. Alain de Libera, Frédéric Nef, «Le discours mystique. Problèmes d'histoire et de méthode ", in Littoral, 9, 1983, p. 80-102, en particulier ici, p. 85 : "On se trouve confronté ici à un véritable problème de méthode. Peut-on faire l'histoire de la mystique sans toucher aucunement à sa tradition? Peut-on comprendre quoi que ce soit de son organisation discursive si l'on néglige les mouvements textuels (entre autres) dont elle s'est ici ou là, au long des siècles, progressivement constituée ? [...] Oui, si l'on a d'avance récusé comme "piété généalogique" toute construction historique faisant place aux notions de tradition et de transmission. " Par souci de vérité, il convient de restituer la phrase de Certeau à laquelle prétendent s'opposer - au nom de la rigueur historique - les auteurs de l'article : "Établir ces cohérences (la corrélation est l'instrument de l'historien), c'est préserver la différence du passé contre la séduction de ressemblances partielles, contre les généralisations que suggère une impatience philosophique ou contre les continuités que postule une piété généalogique " (La Fable mystique I, op. cit., p. 19).

40. Sur cette constitution scientifique, on trouvera dans la Fable mystique II, op. cit., p. 30 et suivantes des considérations épistémologiques très éclairantes.

41. Sur ce point et d'autres, Michel de Certeau a répondu et s'est expliqué dans un entretien avec Mireille Cifali, "Entretien, mystique et psychanalyse ", d'abord paru dans Le Bloc-notes de la psychanalyse, Genève, 4, 1984, p. 135-161, puis dans Espace Temps, 80-81, 2002 ; Michel de Certeau, Histoire/psychanalyse. Mises à l'épreuve, p. 156-175. Au demeurant, la régionalisation caractérise les élaborations scientifiques : que serait une histoire à prétention totalisante ? Que serait une démarche historique qui prétendrait écrire le tout de l'histoire ? Il ne s'agit pas d'évacuer des formations ou des œuvres historiques, mais il faut bien commencer quelque part, ce que Certeau fait en justifiant ce point de départ. Cf. La Fable mystique I, op. cit., p. 29 : « Il a paru préférable de s'installer d'abord au centre de ce champ aux frontières historiques mouvantes et de le considérer dans le moment de sa plus grande formalisation et de sa fin. [...] Des modes de fonctionnement y sont plus lisibles, et donc la détermination d'un lieu, ce qui rend possibles ensuite une histoire régressive de sa formation et une étude de ses avatars ultérieurs. " C’est moi qui souligne. 
pourrait dire épistémologique, mais qui relève aussi d'un respect pour les auteurs dont Michel de Certeau scrute le discours.

Concernant Nicolas de Cues, l'affaire est plus épineuse, car Nicolas critique beaucoup la prétendue suffisance de « la raison naturelle » que revendique explicitement Maître Eckhart. Mais la Fable mystique, précisément, donne les moyens d'analyser plus finement cette affaire en déjouant toute tentative judicatoire d'assigner le Cusain à la place d'un mystique. Un repérage, effectué grâce au Cusanus-portal (outil de recherche qui n'existait pas du vivant de Certeau) ${ }^{42}$, permet de constater que, chez le Cusain, seuls sont employés l'adjectif, un peu plus de trente fois dans l'œuvre philosophique, et l'adverbe mystice, une douzaine de fois. L'adjectif apparaît essentiellement en rapport avec la Théologie mystique du Pseudo-Denys ${ }^{43}$ ou en rapport avec l'Église conçue comme corps mystique $\mathrm{du}$ Christ ${ }^{44}$. On trouve cependant aussi quelques occurrences, plus novatrices, "vis mystica ludi 45 ", " mystica verba 46 ", " mysticam sententiam 47 ", " in mystico sensu 48 ", "in mystico intellectu ${ }^{49}$ ". Et ces occurrences ont l'intérêt de renvoyer à une parole ou à un faire qui semble impliquer un corps. Elles corroborent ainsi l'hypothèse de Michel de Certeau.

Par ailleurs, il y a les lettres aux moines de Tegernsee, en particulier celle du 14 septembre 1453 à l'abbé Gaspard Aindorffer, dans laquelle le Cusain recommande la transposition de la Théologie mystique de Denys mais la défend contre une interprétation strictement affective de la raison qui se transcende ellemême. Il y a aussi le texte de la Visio Dei, au centre de l'interprétation de Certeau, mais il est inutile de lui rapporter la légende d'une éducation chez les Frères de

42. Cf. la base de données accessible à l'adresse internet : cusanus-portal.de. L'ensemble de l'œuvre latine de Nicolas de Cues y est accessible en mode texte, et l'on peut y faire des recherches d'occurrences en contexte.

43. Essentiellement dans la Docta ignorantia, l'Apologia de docta ignorantia, la Visio dei, la Cribatio alchorani, et le De beryllo. On trouve aussi une fois, dans le sermon XVII, une référence à la Théologie mystique de Gerson. À ce propos, cf. Marc Vial, Jean Gerson, théoricien de la théologie mystique, Paris, Vrin, «Études de la philosophie médiévale », 2009. L'auteur, là encore, récuse l'épithète de mystique pour qualifier Gerson, " dans la mesure où elle projette anachroniquement sur le Moyen Âge un terme et des catégories de pensée qui lui sont ultérieurs" .

44. Essentiellement dans la Coniectura de ultimis diebus, la Cribatio alchorani, la Concordantia catholica, l'Epistula ad Rodericum Sancium de Arevalo, la Reformatio generalis, et les sermons. Au sujet du corps mystique du Christ, cf. Michel de Certeau, La Fable mystique I..., op. cit., p. 111. Dans les sermons, par exemple CLXXXIX, 6, on trouve aussi la mention du corps mystique (secret) de la vierge, ou en LXVII, 20 celle du père mystique du Christ (mysticus pater Christi).

45. Nicolas de Cues, Jeu de la boule, 54.

46. Nicolas de Cues, Jeu de la boule, 60.

47. Nicolas de Cues, Jeu de la boule, 61. Il est à noter que ces trois occurrences apparaissent dans une des œuvres les plus tardives du Cusain.

48. Nicolas de Cues, Sermon XIV, 1.

49. Nicolas de Cues, Sermon VIII, 3. 
la vie commune, fondés par Gérard Groote, et de chercher, après celui de la mystique rhénane, le renfort de la Devotia moderna et de la mystique flamande. Je ne dis pas que cela n'existe pas, mais l'absence de traces ${ }^{50}$ devrait conduire à une extrême prudence méthodologique. Il faut en outre souligner qu'à l' "esprit de déploration, d'humilité et de charité ${ }^{51}$ » qui portait ce mouvement s'oppose l'ancrage historique de Nicolas de Cues, prince de l'Église et du Saint-Empire romain germanique, accumulant les prébendes ${ }^{52}$, et ayant une position d'autorité qu'il soutiendra souvent d'un autoritarisme rigoriste, comme dans son évêché de Bressanone ${ }^{53}$ ou à la Curie à Rome ${ }^{54}$.

Alors ? Il faut en revenir au texte, au texte seul, et Michel de Certeau le fait admirablement. D'une certaine manière, il ne laisse rien de côté et multiplie le pointage des opérations de passage dans le texte cusain, du politique au scientifique, du droit au mathématique, du latin à la langue vernaculaire et nationale, de la vision développante à la vision enveloppante, il use mieux que quiconque du vocabulaire cusain, de ses opérateurs, repérant inventions verbales ${ }^{55}$, objets symboliques ${ }^{56}$ et métaphores ${ }^{57}$, montrant comment la pérégrination cusaine défait l'assignation à tout lieu, et comment ce dépouillement conduit, dans l'étourdissement des dissiminations individuelles à la réalisation de l’Un.

Michel de Certeau enchâsse son analyse du De icona dans la considération de l'œuvre en général. Cet opuscule fut écrit pour répondre à une demande des

50. Cf., par exemple, Erich Meuthen, "Cusanus in Deventer ", in Concorda discors. Studi su Niccolo Cusano e l'umanesimo europeo offerti a Giovanni Santinello (éd. Gregorio Paia). Padoue, Editrice Antenore, "Medioevo e umanesimo ", 84, 1993, p. 39-54.

51. Cf. Agnès Minazzoli, "Préface ", in Nicolas de Cues, Le Tableau ou la vision de Dieu, op. cit., p. VII.

52. Cf. Erich Meuthen, "Die Pfründen des Cusanus ", in Mitteilungen und Forschungsbeiträge der Cusanus-Gesellschaft, 1962, p. 16-66.

53. Cf. l'ensemble des articles de Hermann Josef Hallauer rassemblés sous le titre Nikolaus von Kues, Bischhof von Brixen, 1450-1464. Bolzano, Athesia, 2002. Nicolas de Cues aura une activité politique continue depuis le début du Concile de Bâle jusqu'à la fin de sa vie, lorsqu'il est encore chargé de réformer la curie par le Pape Pie II. Mais malgré son expérience de diplomate, le Cusain fit souvent preuve de beaucoup de maladresse, usant et abusant semble-t-il de l'excommunication et des interdits, contrevenant même aux usages de l'époque. $C f$. également mon ouvrage à paraître chez Beauchesne, Nicolas de Cues à l'épreuve du politique.

54. Dans son autobiographie, alors qu'il transcrit dans un style très direct et vivant la plainte que lui adresse le cardinal à propos de la curie, le pape Pie II traite le Cusain de «duri cervicis ", de "tête dure ", "obstinée ". Cf. The commentaries of Pius II, B. VI-IX (trad. Florence Aldan Gragg), Northampton, Leona C. Gabel Smith College Studies in History, 25, 1951, p. 500.

55. Cf. Michel de Certeau, La Fable mystique II, op. cit., p. 260. Ceci étant, si le néologisme que cite Certeau, sur la base de la thèse de Maurice de Gandillac, angelizare, angéliser, est conforme à l'esprit cusain, je n'ai pas réussi à le trouver jusqu'ici dans le texte, même en utilisant le moteur de recherche du Cusanus-portal.

56. Comme le béryl, le jeu de la boule, le tableau, la cuiller... Ibidem, p. 64.

57. Comme celle de la chasse. Ibidem, p. 66. 
moines de Tegernsee qui voulaient mieux comprendre la théorie de la Docte Ignorance. Le traité est accompagné d'un petit tableau, dont le développement montre la coïncidence de la connaissance expérimentale et rationnelle avec la croyance en une ignorance qui ne trouve sa raison que dans la forme d'une théologie circulaire. Michel de Certeau replacera sur son horizon la Cribatio Alchorani ${ }^{58}$ et le De Idiota ${ }^{59}$. La préface propose une expérience, " un exercice spirituel, pratique courante chez les mystiques ". J'ai montré ailleurs ${ }^{60}$ comment l'analyse de Certeau, qui doit beaucoup à la compréhension lacanienne du temps logique, est magistrale car elle permet d'appréhender pratiquement chez le Cusain une théorie hénologique des points de vue, centrale dans son œuvre sur le plan théorétique, épistémologique et pratique.

Cette théorie élabore le rapport à la vérité comme un rapport intersubjectif de croyance, ce qui permet d'éviter le scepticisme le plus radical, là où l'Un est pensé, stricto sensu, dans sa transcendance : "Croire aux dires des autres, c'est ce qui donne accès à une pensée de l'Un ${ }^{61}$ ». La fondation de l'hénologie dépend d'une expérience singulière dans un rapport aux autres, qui permet d'appréhender, ou plus exactement de construire un espace qui, tout en requérant une perception sensible, se découvre comme un espace structural, un espace de coexistence et de colocation, dans lequel les différences n'apparaissent pas aprèscoup comme des relations externes, entre des identités préexistantes, mais comme des « relations constitutives de déterminations réciproques".

Par là se comprend aussi la coessentialité de l'autre pour chacun : sans celuici, le monde qui se dévoile est insensé ; c'est donc, par un retournement typiquement cusain, la croyance elle-même qui se dédouble, en croyance insensée et croyance sensée : là, croyance et raison coïncident. Cette folie en évite une autre, irrémédiable ${ }^{62}$. Dans la lettre du 22 septembre 1452 à Gaspard Aindorffer, Nicolas le dit bien : il faut aussi se méfier de l'affectus :

«Si nous rencontrons [...] un homme simple qui observe avec zèle les commandements de Dieu, nous savons qu'il est aussi un fidèle chrétien, et nous croyons que, tel Paul, il peut être ravi jusqu'à la vision ; mais dans le ravissement beaucoup sont trompés, qui s'attachent aux images et jugent vraie une vision imaginaire. La vérité est objet de l'intellect ${ }^{63}$."

58. Michel de Certeau, La Fable mystique II, op. cit., p. 104.

59. Ibidem, p. 112 sq.

60. Jocelyne Sfez, « De la docte ignorance. Perspectives et foi chez Cues et Lacan », art. cité.

61. Michel de Certeau, La Fable mystique II, op. cit., p. 119.

62. "Cette folie première rend possible la théorie " (Michel de Certeau, La Fable mystique II..., op. cit, p. 119. Toute proportion gardée, on n'est pas si loin que cela d'un pari, en un sens pascalien, c'est-à-dire un pari éminemment rationnel.

63. Nicolas de Cues, Lettre à Gaspard Aindorffer du 22 septembre 1452, in Nicolas de Cues, Lettres..., op. cit., p. 25. 
Nicolas de Cues, dans sa fonction épiscopale, a ainsi toujours montré la plus grande circonspection pour ce qui se donnait comme miraculeux et il a notamment interdit le culte des hosties sanglantes ${ }^{64}$. Certes, la préface du De icona débouche sur l'acte de croire, mais pas n'importe lequel, il emporte avec lui la positivité de l'autre, au point que l'Un peut être appréhendé comme tel sans déchoir de sa transcendance.

La préface ne conduit pas non plus au vide et au silence, peut-être - mais je n'en suis pas convaincue - à la prolifération du texte ${ }^{65}$. Celui-ci ne s'emballe pas, et si Nicolas n'arrêtera jamais sa quête, sa chasse, son texte reste étonnamment réglé, presque toujours dialogique. Toute mention d'une folie, que pointe systématiquement Certeau, est aussi immédiatement reprise pour faire langue, saisir ce qu'il y a de sensé. Si la préface joue sur le double registre d'un « exercice spirituel » et d'une expérience scientifique, elle ne suppose donc pas l'abandon de l'esprit, mais le passage de la raison à l'intelligence, en même temps que de la visio sensibilis à la visio mentalis ou intelligibilis, comme du solipsisme à la communauté. Le face-à-face, la vision de face, reste intenable, insoutenable, seulement entraperçu et fondé finalement a posteriori dans un tiers.

À cet égard encore, le texte cusain est clair : avec circonspection, Nicolas exprime sans ambiguïté qu'il n'a jamais été ravi comme Paul, il n'a jamais connu l'extase. L'exercice spirituel ne conduit pas factuellement à une expérience mystique et il a une autre valeur. $\mathrm{Si}$ "science expérimentale» il y a bien chez le Cusain - le terme y est expressément dans le quatrième dialogue du De idiota ce n'est pas au sens de Jean-Joseph Surin, mais de Roger Bacon. La Venatio sapientae cusaine conduira à une venatio scientiarum, chez Van Helmont par exemple, dans toute leur positivité. Cela oblige aussi à reconsidérer le hors-texte de la péroraison dans la Docte Ignorance, dans lequel Nicolas de Cues parle de la coïncidence des opposés comme d'un don reçu du Père des Lumières. D'abord, c'est précisément un hors-texte, ce qui signifie que le texte même de la Docte Ignorance se tient sans sa condition. Ensuite, ce don ne résulte pas d'un ravissement, Nicolas le soulignera dans le De icona. Enfin, même ce texte ultérieur ne

64. Cf., par exemple, Jutta Fliege, « Nikolaus von Kues und der Kampf gegen das Wilsnacker Wunderblut", in Das Buch als Quelle historischer Forschung (éd. Joachim Dietze), Munich, Verlag Dokumentation, 1978, p. 62-70; Morichi Watanabe «The German Church shortly before the Reformation: Nicolaus Cusanus and the Veneration of the Bleeding Hosts at Wilsnack ", in Reform and Renewal in the Middle Age and the Renaissance (éd. Thomas Izbicki, Christopher Bellito), Leyde, Brill, 2000, p. 210-223 ; Nikolaus Staubach, "Cusanus laudes. Nikolaus von Kues und die Devotia moderna im spätmittelalterischen Reformdiskurs ", in Frühmittelalterliche Studien, De Gruyter, 34, 2010, p. 259-337.

65. Il est vrai que chaque texte cusain constitue une tentative d'approche du nom divin qui, comme le note constamment Nicolas, ne peut qu'échouer. Mais il affirme aussi, et dès la Docte ignorance, que chaque échec, en tant qu'il est une tentative, s'ajoute comme geste aux tentatives et échecs précédents et contribue ainsi à une approche conjecturelle indéfinie du Divin. En ce sens, le discours s'infinitise lui-même, cela ne veut pas encore dire qu'il se défait et prolifère. 
fut pas si convaincant que cela : les moines de Tegernsee ne cessèrent de demander d'autres explications. Nicolas de Cues accédera une fois encore à leur requête en envoyant le De beryllo. Dans cet opuscule de 1458, nourri notamment par la relecture d'Aristote, le concept de vision intellectuelle est exposé dans son plein développement, en recourant systématiquement à la pratique mathématique, qui implique l'exercice de la seule pensée réflexive, accessible à tout un chacun qui en fait l'effort.

Il est évidemment impossible ici de rendre justice au texte de Michel de Certeau, dont je n'ai pas, loin de là, abordé toute la richesse et ce qu'il donne à penser. Il a l'immense mérite de présenter une lecture complète et précise du Cusain, informée de la culture et des enjeux politiques et ecclésiologiques de son temps. La lecture de Certeau produit un effet sidérant de vérité lorsqu'elle s'attache au détail du texte de la préface du De icona qu'elle relie aux autres textes. Elle me semble surtout poser à nouveaux frais le problème du rapport de Nicolas de Cues à la mystique en examinant concrètement ses gestes et ses procédures de pensée. Certes, comme le dit Michel de Certeau, «la littérature mystique ne commence pas au XVI ${ }^{e}$ siècle, même si elle ne se découpe un nom et une formalité propres que pendant la seconde moitié de ce siècle ${ }^{66}$ ». La mystique rhénane et la mystique flamande ont déjà acquis depuis longtemps leurs lettres de noblesse. Comme Michel de Certeau le montre, Nicolas y a quelque affinité, sans pour autant qu'il faille faire de lui un de leurs tenants ou leur héritier. En particulier, le De icona est assurément important pour les spirituels et les mystiques au XVII ${ }^{\mathrm{e}}$ siècle. Cela ne fait pas pour autant du Cusain un mystique : «La vérité, affirme-t-il, est l'objet de l'intellect. » Sa pratique institutionnelle et d'autres de ses textes s'y opposent. La force du texte de Michel de Certeau consiste précisément à ne pas assigner le Cusain à cette place tout en montrant la fécondité de sa pensée pour le discours mystique.

Jocelyne SFEZ

ERIAC-Université de Rouen jocelyne.sfez@lycos.com

66. Michel de Certeau, La Fable mystique I, op. cit., p. 47. 
